Accelerator Division

Alternating Gradient Synchrotron Department

BROOKHAVEN NATIONAL LABORATORY

Upton, New York 11973

Accelerator Division

Technical Note

AGS/AD/Tech. Note No. 340

\title{
Modeling the QN17 Correction Scheme with the BEAM Program
}

\author{
C. J. Gardner
}

July 6,1990

The QN17 correction scheme, used to correct the $2 Q_{H}=17$ and $2 Q_{V}=17$ resonances in the AGS, is discussed in references [1-3]. This scheme has been put into a modified version of the BEAM code so that it's effect on the machine can be modeled. The modified BEAM code also contains the nuquads, which are used to adjust the tunes of the machine at low field, and the beta quads, which are used to eliminate the beta function distortion due to remanent fields. Following are results obtained with this modified code.

\section{Calibrations Used in the Modified BEAM Code}

The four 'knobs', COS $17 \mathrm{X}$, SIN $17 \mathrm{X}$, COS $17 \mathrm{Y}$, and SIN $17 \mathrm{Y}$, used in the QN17 correction scheme are labeled CX, SX, CY, and SY in the modified BEAM code. For given settings of these knobs, the 16 quadrupoles used in the scheme are excited with currents according to the formulae of reference [3].

The horizontal and vertical nuquads [1] are excited with currents determined by the settings of NUQH and NUQV. A command of $N$ counts issued to NUQH or NUQV results in a current of $N / 80$ amps in the corresponding nuquad string.

A command of $N$ counts issued to BTQD results in a current of $N / 500$ amps in the beta quads according to the scheme of reference [1]. 


\section{Results}

The modified BEAM code was run with the 250 gauss field map option. The momentum on the reference orbit for this field map is $0.650 \mathrm{GeV} / \mathrm{c}$.

Table I lists the tunes, $Q_{H}$ and $Q_{V}$, obtained by the code for various settings of CX, NUQH, NUQV, and BTQD. (SX, CY, SY were all set to zero.)

\begin{tabular}{|c|c|c|c|c|c|}
\hline \multicolumn{7}{|c|}{ Table I } \\
\hline CX & NUQH & NUQV & BTQD & $Q_{H}$ & $Q_{V}$ \\
\hline 0 & 0 & 0 & 0 & 8.7294 & 8.7652 \\
0 & 0 & 0 & 2800 & 8.7430 & 8.7759 \\
500 & 0 & 0 & 2800 & 8.7428 & 8.7759 \\
1000 & 0 & 0 & 2800 & 8.7422 & 8.7760 \\
0 & 1309 & -654 & 2800 & 8.5000 & 8.7659 \\
\hline
\end{tabular}

The first row of the table gives the bare machine tunes and the second row gives the tunes with the beta function distortion corrected. The third and fourth rows show that the setting of CX has only a small effect on the tunes as long as they are sufficiently far from the $2 Q_{H}=17$ resonance stopband. Note that with the QN17 correction scheme turned off-i.e. with $\mathrm{CX}=\mathrm{SX}=\mathrm{CY}=\mathrm{SY}=0$-there are no quadrupole field configurations in the beam code which can excite the $2 Q_{H}=17$ resonance. The nuquads can therefore be adjusted so that $Q_{H}=8.5$ as indicated in the last row of Table I.

If the QN17 corrections are turned on, then quadrupole field configurations are created which distort the beta function and will excite the $2 Q_{H}=17$ resonance whenever the (unperturbed) horizontal tune is inside the resonance stopband. To determine the width of the stopband, the QN17 corrections were turned on and the range of settings of NUQH was found for which the absolute value of the trace of the transfer matrix for one turn around the machine was greater than two. The data of Table II were obtained with $\mathrm{SX}=\mathrm{CY}=\mathrm{SY}=0, \mathrm{BTQD}=2800$, and NUQV $=-654$. Under these conditions the BEAM code was run for various settings of NUQH. The horizontal tines obtained with $\mathrm{CX}=500$ and $\mathrm{CX}=0$ are listed in the second and third columns respectively. With $\mathrm{CX}=500$ the absolute value of the trace of the transfer matrix is greater than two for settings of NUQH between 1265 and 1353. With the QN17 corrections 
turned off, this range of settings corresponds to tunes between 8.4893 and 8.5108. The width of the stopband opened up when $\mathrm{CX}=500$ is therefore $8.5108-8.4893=0.0215$.

\begin{tabular}{|c|c|c|c|}
\hline \multicolumn{4}{|c|}{ Table II } \\
\hline \multirow{2}{*}{ NUQH } & \multicolumn{2}{|c|}{ Horz Tune } & Horz Beta (m) \\
\cline { 2 - 4 } & CX = 500 & CX $=0$ & CX $=500$ \\
\hline 1220 & 8.5189 & 8.5218 & 38 \\
1230 & 8.5160 & 8.5194 & 43 \\
1240 & 8.5130 & 8.5169 & 48 \\
1250 & 8.5096 & 8.5145 & 56 \\
1260 & 8.5053 & 8.5120 & 97 \\
1261 & 8.5047 & 8.5118 & 104 \\
1262 & 8.5040 & 8.5115 & 112 \\
1263 & 8.5033 & 8.5113 & 150 \\
1264 & 8.5023 & 8.5111 & 200 \\
1265 & 8.5004 & 8.5108 & \\
& Stopband & & \\
1353 & 8.4979 & 8.4893 & \\
1354 & 8.4968 & 8.4890 & 160 \\
\hline
\end{tabular}

The stopband width can also be calculated using perturbation theory. Using equations (5) and (7) of reference [2], and equation (29) of reference [3], one finds that the stopband opened up when $\mathrm{CX}=M$, $\mathrm{SX}=\mathrm{CY}=\mathrm{SY}=0$, has width

$$
W=\frac{2}{\pi}\left(\frac{e Q}{c P}\right)\left(R^{2}-1\right) b \frac{M}{1000},
$$

where $Q$ is the integrated strength of each quadrupole used in the QN17 correction scheme, $P$ is the momentum, $R=B / b$, and $B$ and $b$ are the AGS lattice beta maximum and minimum. Inserting the numbers $Q=41.08$ gauss, $c P / e=3335641$ gauss-cm per $\mathrm{Gev}, B=2169 \mathrm{~cm}$, and $b=1046 \mathrm{~cm}$, we find

$$
W=\frac{0.0271}{P}\left(\frac{M}{1000}\right)
$$

where $P$ is the momentum in $\mathrm{GeV} / \mathrm{c}$. With $M=500$ and $P=0.650$ $\mathrm{GeV} / \mathrm{c}$ the stopband width is then 0.0208 which is in good agreement with the value obtained from the beam code. 
The perturbed tunes listed in Table II-i.e. those obtained with $\mathrm{CX}=500$-can also be calculated using perturbation theory. Using equation (2.27) of reference [4] one obtains values which agree with those obtained by the BEAM code.

The right-hand column in Table II lists the maximum values of the horizontal beta function obtained with $\mathrm{CX}=500$. (The maximum beta obtained with the QN17 corrections turned off is approximately 22 meters.) Here we see-as expected-that the beta function distortion increases (and in fact becomes infinite) as one approaches the stopband. The perturbation theory calculation of the beta function distortion is discussed in reference [4]. Using equation (2.55) of this reference one obtains results in agreement with those obtained by the BEAM code.

\section{References}

1. C. J. Gardner, 'A Review of the Low-field Correction System Presently Employed in the AGS', AGS/AD/Op. Note No. 17, February 4, 1988.

2. C. J. Gardner, 'Effective Placement of Stopband Correction Elements in an AGS Lattice', AGS/AD/Tech. Note No. 321-Revised, September 29, 1989.

3. C. J. Gardner, 'AGS Stopband Correction Schemes: Some Useful Numbers and Relations', AGS/AD/Tech. Note No. 335, March 8, 1990.

4. J. C. Herrera and M. Month, "Theory of Gradient Stopbands in the AGS', BNL Report No. 11964. 